\title{
Medical Professionalism Is Like Pornography: You Know it When You See it
}

\section{J. Brantley Thrasher, MD, FACS, Cynthia R. Hamady, and Lindsay W. Franklin}

Addressing professionalism is a key role of Certification Boards, but how best to do this is not clear. This article describes a $360^{\circ}$ approach to monitoring and enhancing professionalism taken by the American Board of Urology (ABU). In addition to monitoring full and active medical licenses, ABU has identified ethical issues specific to Urology, includes a position article on ethics in Urology on its Web site, and requires a completion of modules on ethics. As a part of its 10-year cycle, the Board requires peer evaluations from other urologists in the community. Finally, and most importantly, ABU uses a portfolio practice log to evaluate the candidates' use of procedures appropriate to their stated subspecialty area of expertise, evaluation of potential overuse or inappropriate use of procedures and a narrative that details any major complications associated with their procedures. ( $\mathrm{J}$ Am Board Fam Med 2020;33:S62-S64.)

Keywords: Certification, Licensure, Professionalism, Urology

Medical professionalism is the basis for our social contract with the public - the safety of which is our reason for existence. However, physicians today find themselves in an era of increasingly integrated health care systems and hospital employment that incentivizes work productivity, sometimes at the expense of research, education, and even professional and ethical conduct.

We all recognize that for us to continue to earn public trust in professional self regulation, medical professionalism must be taught to our young physicians in training and monitored longitudinally for evidence to the contrary. Monitoring and assessing professionalism—our goal and a recent premise of the Commission Report ${ }^{1}$ can be a difficult task requiring a multi-faceted approach.

The American Board of Urology (ABU) recognizes the importance of monitoring and evaluating medical professionalism and the difficulties inherent to defining the behavior. As Supreme Court Justice Potter Stewart stated in trying to define

This article was externally peer reviewed.

Submitted 4 November 2019; revised 14 May 2020; accepted 19 May 2020.

From the American Board of Urology, Charlottesville, VA.

Funding: None.

Disclosures: None.

Corresponding author: J. Brantley Thrasher, MD, The American Board of Urology, 600 Peter Jefferson Pkwy \#150, Charlottesville, VA 22911 (E-mail: brant@abu.org). pornography, it was difficult to provide a shorthand description. However, he noted, "But I know it when I see it...." The definition of professionalism has plagued all Member Boards of the American Board of Medical Specialties (ABMS) but we all recognize the need to demand ethical and professional conduct from our Diplomates.

The ABU currently has 7860 time-limited Diplomates and 3160 time-unlimited Diplomates. Affirming primacy of patient welfare, patient autonomy and social justice - the principles elaborated in the 2002 Physician Charter on Medical Professionalism ${ }^{4}$ - the Board addressed a growing concern: American urologists treating patients in other countries with high-intensity focused ultrasound, though the technology was not Food and Drug Administration (FDA) approved in the United States at the time. In a 2008 ABU Newsletter article titled, "Let us Focus on Professionalism and Ethics in Urology," the Board of Trustees tackled this untoward practice head on. These widely publicized cases of urologists potentially placing financial benefit over patient safety became an inflection point for the $\mathrm{ABU}$ and its quest to promote ethical and professional behavior from all certified diplomates.

The ABU assesses medical professionalism and ethical practice through a variety of mechanisms at periodic levels in a Diplomate's continuing 
certification cycle. Since 1985, ABU awarded certificates are time-limited 10 years, and from 2009 onward subject to maintenance through Diplomate evaluation. Life Long Learning is composed of 2 levels. At both levels, Diplomates are required to provide a detailed explanation of any professional lawsuits or arbitration, loss of privileges, license restrictions, or substance abuse treatment occurring over the past 5 years. During Level 1 (years 2 to 5), Diplomates are required to complete Patient Safety Videos (PSVs) covering public reporting, just culture, communication and disruptive behavior. In addition, a Professionalism and Ethics Module (PEM) consisting of defining professionalism, behaviors that undermine a culture of safety, barriers to addressing these behaviors and guiding principles for interventions is also required. This module also discusses the accountability pyramid adapted from Hickson et al. ${ }^{3}$ Peer review, another important tool in this process, is also solicited from colleagues, partners and/or other urologists, nurses, and administrators. Input from other sources such as newspaper clippings, reports from the Discipline Associations Network for Sustainability and National Practitioners Database, and at times, phone interviews with concerned parties are also received. The ABU finds cross-sectional peer review to be critical in the overall assessment of ethical conduct and professionalism and generally starts with the chiefs of staff, urology, surgery, and anesthesia at each facility where the Diplomate performs at least 50 cases annually. Because parties, especially protective partners or colleagues, are often reluctant to answer questions on whether the applicant has acceptable ethical standards or exceptional professional attributes, it is sometimes necessary to probe further by extending the pool of reviewers. When undertaken, such measures uncover unethical or questionable professional behavior. To date, 1224 time-limited Diplomates have completed Level 1 PEMs and 699 Diplomates have completed all PSVs.

In Level 2 (years 7 to 9), Diplomates complete at least 1 Patient Safety Module from a menu of 10 choices including abbreviations and symbols, communication-system based practices, disruptive behavior, safety during surgery, fatigue and patient safety, informed consent (including issues such as patient comprehension and patient's autonomy to make the choice), patient handoff and preventing medical errors, proper relationship with industry, and finally, sexual misconduct. A test is required after each module. At this level, Diplomates also undergo peer review again and are required to submit a description of their practice and the population demographics served, as well as a breakdown chart of hours typically worked per week in administration, Operating Room, research, and clinic.

However, one of the most valuable requirements of Level 2 is the 6-month practice $\log$, which gives an excellent overview of the Diplomate's surgical and office practice compared with a peer group of approximately 600 other applicants. For statistical analysis and proper comparisons, practice logs must be submitted in a prescribed format and must include everything for which the Diplomate has billed within the 6-month period selected from a 15 -month window leading up to January 31 of the submission year. Once received, the logs are then annualized and compared in every category to the logs of all other applicants in the recertification class (generally 550 to 600 per cycle). These logs allow us to assess whether or not a Diplomate is over using certain tests, following our published guidelines for standard practice, using procedures that are outdated or erroneous, experiencing a large number of Clavien Grade III or greater complications, choosing a testing module inappropriate to their current practice, or experiencing an unusually high number of professional liability suits. Narratives of Clavien Grade III or higher complications coinciding with the log must accompany the submission, as well as narratives of any 30-day postoperative mortalities experienced within the past 10 years.

A Board committee reviews all logs and any concerns are outlined in a letter back to the Diplomate. Since 2015 , letters are mailed to approximately $20 \%$ of Diplomates requesting further information on the use of certain Current Procedural Terminology (CPT) codes and clarifications of reported complications or lawsuits. If warranted by the Diplomate's response, deidentified charts of a number of relevant patients are requested and reviewed to additionally assess appropriate treatment and usage of CPT codes in question. Explanations received that are deemed inappropriate, outside standard practice or potentially dangerous can result in a variety of actions such as a request for a personal interview with the Board, requirements to complete and document a designated number of Category 1 Continuing Medical Education (CME) credit hours 
in a specified area, and/or a visit to the applicant's practice setting by 1 or more representatives of the Board. Examples of such issues are logs that identify the use of outdated procedures such as female urethral dilations, inappropriate diagnostic procedures such as bilateral ureteroscopy for all patients experiencing hematuria, overuse of ultrasound, or an inordinate number of serious complication resulting from surgeries.

Log reviews have been and continue to be one of the most valuable tools to assess cost-effective ethical standards of conduct in the field of urology. In many cases, simply identifying a lack of standard of care practice has yielded quick results of significant practice modifications and apologetic letters from Diplomates. Identification of miscoded or overused codes has been a teaching experience for many Diplomates unaware that they were practicing well outside their peer group. Finally, in some cases, the Board has uncovered notable nefarious breaches in ethical behavior which have resulted in suspension or revocation of an applicant's certificate.

In summary, the Trustees of the ABU recognize that medical professionalism requires a urologist to uphold a set of qualified standards leading to continued public confidence and trust. It is the social contract that we have with the public we serve. However, assessing professionalism can be difficult, requiring a multi-faceted approach. Through the use of learning modules, peer review, CME, complications narratives and practice logs, we continue to assess professional and ethical conduct and when absent, take the necessary steps to correct the behavior.

To see this article online, please go to: http://jabfm.org/content/ 33/Supplement/S62.full.

\section{References}

1. Colenda CC, Scanlon WJ, Hawkins RE. Vision for the future of continuing board certification. JAMA 2019;321:2279-80.

2. Jacobellis v. Ohio | US Law | LII/Legal Information Institute. Available from: https://www.law.cornell. edu/supremecourt/text/378/184. Accessed September 13, 2019.

3. Medical professionalism in the new millennium: a physician charter. Ann Intern Med 2002;136:243-6.

4. Vanderbilt University Medical Center. Professionalism pyramid. Available from: https://ww2.mc.vanderbilt. edu/cppa/45627. Accessed September 13, 2019. 